\title{
Advances in the diagnosis and management of pulmonary aspergillosis
}

\begin{abstract}
Aspergillus is a mould that is ubiquitous in nature and may lead to a variety of infectious and allergic diseases depending on the host's immune status or pulmonary structure. Invasive pulmonary aspergillosis occurs primarily in patients with severe immunodeficiency. The significance of this infection has dramatically increased with growing numbers of patients with impaired immune state associated with the management of malignancy, organ transplantation, autoimmune and inflammatory conditions; critically ill patients appear to be at an increased risk as well. The introduction of new noninvasive tests, combined with more effective and better-tolerated antifungal agents, has resulted in lower mortality rates associated with this infection. Chronic pulmonary aspergillosis is a locally invasive disease described in patients with chronic lung disease or mild immunodeficiency. Recently, the European Society for Clinical Microbiology and Infectious Diseases provided a more robust sub-classification of this entity that allows for a straightforward approach to diagnosis and management. Allergic bronchopulmonary aspergillosis, a hypersensitivity reaction to Aspergillus antigens, is generally seen in patients with atopy, asthma or cystic fibrosis. This review provides an update on the evolving epidemiology and risk factors of the major manifestations of Aspergillus lung disease and the clinical manifestations that should prompt the clinician to consider these conditions. It also details the role of noninvasive tests in the diagnosis of Aspergillus related lung diseases and advances in the management of these disorders.
\end{abstract}

Key words: asperilus, invasive pulmonary aspergillosis

Adv Respir Med. 2019; 87: 231-243

\section{Introduction}

Aspergillosis refers to a variety of conditions caused by aspergillus spp. - a ubiquitous saprophytic mold commonly found in soil, water, plants, and building materials [1]. First recognized in Florence in 1729, transmission is via inhalation of fungal spores, and mostly affects immunocompromised individuals or those immunocompetent individuals with underlying chronic lung diseases [2]. A. fumigatus accounts for most human infections, followed by $A$. flavus, $A$. terreus, $A$. niger, and A. nidulans [3]. Aspergillosis can cause a spectrum of pulmonary disease depending the characteristics of the individual host: 1) invasive pulmonary aspergillosis; 2) chronic pulmonary aspergillosis; and 3) allergic bronchopulmonary aspergillosis.

\section{Invasive pulmonary aspergillosis}

Invasive pulmonary aspergillosis (IPA) is the most severe on the spectrum of diseases caused by Aspergillus spp. It mostly affects the immunocompromised individuals, and carries a high mortality rate ranging from $38 \%$ to $75 \%$ with high economic burden [4-9]. Despite advances in medical therapy, incidences of IPA have increased remarkably, owing to more aggressive chemotherapy regimens for hematologic malignancies, increasing number of solid organ transplants, and expanding use of immunosuppressants [10]. In two large studies examining autopsy data between 1978 to 1992, and 1993 to 2005 , the incidence of aspergillus as the causative organism increased from $17 \%$ to $60 \%$ from 1978 to 1992, and this remained stable between 1993 to 2005. Despite changes in epidemiology,

Address for correspondence: Ayman 0. Soubani, Division of Pulmonary, Critical Care and Sleep Medicine, Wayne State University School of Medicine, Detroit, USA e-mail: asoubani@med.wayne.edul

DOI: 10.5603/ARM.2019.0061

Received: 10.10.2019

Copyright (C) 2019 PTChP

ISSN 2451-4934 
aspergillus remained as the most common fungal pathogen [11, 12].

IPA is characterized by the invasion of pulmonary arterioles and lung parenchyma by Aspergillus hyphae that leads to ischemic necrosis, intravascular thrombosis, hemorrhagic infarct from rapid hyphal growth [13, 14]. The angioinvasion of distant organs via hematogenous dissemination frequently involves the kidneys, liver, spleen, and the central nervous system.

\section{Risk factors}

At risk patient populations have classically included immunocompromised individuals such as recipients of allogeneic hematopoietic stem cell transplantation (HSCT), solid organ transplant (SOT) recipients, patient on prolonged corticosteroids, individuals with advanced AIDS and chronic granulomatous disease [15].

Inhalation of aspergillus spores are common, and in most immunocompetent hosts, mechanisms are in place to prevent its germination and growth. Respiratory epithelial cells and mucociliary clearance act as first line of defense against inhaled aspergillus species [16]. Alveolar macrophages activation by NADPH oxidase activation contributes to both conidial clearance and secretion of inflammatory mediators, resulting in recruitment of neutrophils and activation of cellular immunity. Neutrophils play an important role, via NADPH oxidase dependent pathway in inducing hyphal damage; and via a NADPH oxidase independent pathway to inhibit conidial growth [16]. Highlighted in a study over 30 years ago, prolonged neutropenia has been a classic risk factor for IPA, with both the severity as well as the duration of neutropenia as contributing factors for risk of IPA [17]. It was estimated that patients with granulocytopenia developed signs of IPA at a rate of $1 \%$ per day in the early phase, and approximately $4.3 \%$ per day between days 24-36 [17].

More recent years, IPA has been increasingly reported in non-neutropenic, immunocompetent but critically ill patients such as those with severe influenza A and B $[18,19]$ liver disease or those with COPD exacerbation who received systemic and or inhaled corticosteroids [20]. One multicenter cohort study found influenza to be independently associated with IPA (95\% CI 2.63-10.26; $\mathrm{p}<0.0001$ ), and such patients had a $\sim 51 \%$ mortality rate [21]. Incidence rate of IPA is as high as $16 \%$ in patients with alcoholic liver cirrhosis [22]. The pathogenesis of IPA in these patients tends to be nonangioinvasive, and there is an exacerbated inflammatory environment resulting in tissue injury [14].

\section{Clinical presentation}

Early symptoms can be non-specific, including dry cough and fever, and pleuritic or non-specific chest pain. Immunocompromised patients often do not have fever due to their inability to mount an adequate inflammatory response. Progression to pneumonia occurs within 1-2 days [13]. Angioinvasive IPA predominates in neutropenic patients, with rapid progression and clinical deterioration occurring over hours to days [23]. Hemoptysis is suggestive of pulmonary infarction, and sometimes massive hemoptysis is seen in patient with formation of cavitation during neutrophil recovery phase.

\section{Diagnosis}

Diagnosing IPA is difficult in patients with known risk factors. Diagnosing IPA in those critically ill patient without classic risk factors can be even more challenging, as studies have found IPA to be frequently misdiagnosed based on autopsy [20,24].

The gold standard for diagnosis is via biopsy of lung tissue, in combination with positive culture of Aspergillus spp. from the biopsy specimen. However, patients with suspected IPA often have profound thrombocytopenia, or are clinically unstable to undergo biopsy procedures. Focus has been placed on noninvasive biochemical markers that allows for rapid and reliable diagnosis of IPA.

Galactomannan (GM) is part of the cell wall of aspergillus that is released during fungal growth[13]. The use of serum and bronchoalveolar lavage (BAL) GM is recommended by the Infections Diseases Society of America (IDSA) as a diagnostic marker for IPA in both adult and pediatric patients with hematological malignancy or recipient of allogeneic HSCT, with a sensitivity of $\sim 70 \%$ [15]. However, its use is population specific, and sensitivity decreases in nonneutropenic patients, CGD patients, and those with SOT to as low as $20 \%$. Furthermore, obtaining serum GM is not recommended in patients receiving antifungal therapy or prophylaxis, but rather should be obtained from BAL specimen. A Cochrane meta-analysis by Leeflang et al. conducted in immunocompromised patients demonstrated serum GM to have sensitivity varying between $78 \%$ to $63 \%$ and specificity of $85 \%$ to $93 \%$, depending on the optical density index (ODI) used as cut off from 0.5 to 1.5 re- 
spectively [25]. Similar study on GM obtained from BAL fluid showed similar trend and variability in sensitivity and specificity based on ODI used, with a decrease in sensitivity from $88 \%$ $(75-100 \%, 95 \% \mathrm{CI})$ to $78 \%(61-95 \%, 95 \% \mathrm{CI})$, and increase in specificity from $81 \%$ (71-91\%, $95 \% \mathrm{CI})$ to $93 \%(87-98 \%, 95 \% \mathrm{CI})$ when a ODI of 0.5 and 1 was compared [26]. Further increasing ODI to 1.5 significantly decreased variability of specificity, and appeared as a strong indicator of IPA. This increase in false negative rate and decrease in false positive rate with increasing ODI value should be taken into account when using GM as a diagnostic marker. A more recent systemic review and meta-analysis by Haydour and colleagues demonstrated serum GM had moderate sensitivity of $71 \%(64-78 \%, 95 \% \mathrm{CI})$ and high specificity of $89 \%$ (84-92\%, 95\% CI) [27]. ODI of 1 was considered to provide optimal sensitivity [79\%, $(60-91 \%, 95 \% \mathrm{CI})]$ and specificity $(88 \%, 78-94 \%, 95 \%$ CI) [28]. Increased diagnostic accuracy was again demonstrated in BAL GM with overall sensitivity and specificity of $84 \%(73-91 \%, 95 \% \mathrm{CI})$ and $88 \%(81-91 \%$, $95 \% \mathrm{CI})$ respectively [27].

Beta-D-glucan (BDG) is another cell wall polysaccharide found on the cell walls of most fungi. A meta-analysis by Hou et al. included 1068 patients from 11 studies found the sensitivity and specificity of the diagnostic accuracy of serum BDG to be $75 \%$ (64-84\%, 95\% CI) and $87 \%$ (81-92\%, 95\% CI) respectively as a screening tool for invasive fungal disease [29]. Furthermore, the pooled sensitivity and specificity for IPA was 73\% (62-86\%, 95\% CI) and 81\% (64-85\%, 95\% CI) respectively [29]. The various type of BDG assays available, with different cutoffs for BDG levels created potential for error. Combining serum GM with serum beta-D-glucan (BDG) or having two positive BDG test consecutively significantly increases specificity [30].

Aspergillus PCR is more sensitive than respiratory cultures, with a sensitivity and specificity of $79.2 \%(71-85.5 \%, 95 \% \mathrm{CI})$ and $79.6 \%$ (69.9-86.6\%, 95\% CI) respectively [31]. When two positive PCR results are required, sensitivity and specificity were $59.6 \%$ (40.7-76.0\%, 95\% CI) and $95.1 \%$ (87.0-98.2\%, 95\% CI). The increased specificity in two consecutive tests indicate value of using PCR as a diagnostic screening tool. Avni et al. demonstrated higher summary sensitivity and specificity of BAL PCR of 90\% (77-96\%, 95\% CI) and 96\% (93-98\%, 95\% CI) respectively [32]. When including only studies adhering to European Organization for Research and Treat- ment of Cancer and the Mycoses Study Group (EORTC/MSG) diagnostic criteria, sensitivity and specificity decreased to $77 \%$ and $93 \%$. Study also found reduced sensitivity of BAL PCR in patients receiving antifungal treatment or prophylaxis. The sensitivity of aspergillus PCR on BAL fluid was higher, though there are instances of lowered specificity that may be attributed to the fact that aspergillus often colonized in the lungs [33]. Due to lack of standardization and validation, the Aspergillus PCR has not been cleared by the FDA for clinical use in the United States [15]. The mycological tests used for diagnosis are summarized in Table 1.

Radiographic imaging is frequently used to aid in diagnosis of IPA. HRCT is the preferred method at the early stages to identify lesions of IPA, with features including nodules, consolidative lesions, and wedge-shaped infarcts. Characteristic feature of IPA, especially in neutropenic patients, is the 'halo sign', which is a central nodule that's $>1 \mathrm{~cm}$ in diameter surrounded by a ground-glass opacity reflecting hemorrhage [34] (Figure 1). Halo sign is seen only during the early stages of angioinvasion, and thus can be easily missed if CT scan is not done during this window. The 'air crescent sign' seen in $\sim 50 \%$ of cases usually two weeks after disease progression, after the patient's immune system has recovered from the initial insult. It is a crescentic area of radiolucency within the nodular opacity [34]. This represents the retracted and infarcted lung, and its presence may be too late to be helpful in making the diagnosis. CT findings in tracheobronchitis typically show thickened tracheobronchial wall with presence of centrilobular micronodules arranged in a 'tree-in bud' pattern [34]. With increasing incidence of IPA in non-neutropenic patients, various CT image findings exist, with no typical findings described yet [35].

Based on the advancement in diagnostic tests and imaging studies, the European Organization for the Research and Treatment of Cancer/Mycoses Study Group (EORTC/MSG) consensus group revised its standard definition for IPA in 2008 summarized in Table 2 [36].

Most recently, ATS guidelines in 2019 strongly recommended the use of serum GM in severely immunocompromised patients with unexplained lung infiltrates suspected of IPA [37]. BAL-GM testing is strongly recommended in those patients with negative serum-GM but have strong risk factors of IPA, or those with positive serum-GM but have confounding factors for false-positive results [37]. 
Table 1. Mycological tests for diagnosis of IPA

\begin{tabular}{|c|c|c|c|}
\hline & Sensitivity/specificity (95\% CI) & LR (95\% CI)/ PPV/NPV & ATS recommendations \\
\hline Serum GM & $\begin{array}{l}\text { Sensitivity: } 79 \%(60-91 \%) \\
\text { Specificity: } 88 \%(78-94 \%)\end{array}$ & $\begin{array}{l}\text { Positive LR: } 6.6(3.4-12.5) \\
\text { Negative LR: } 0.24(0.11-0.50)\end{array}$ & $\begin{array}{l}\text { Strong recommendation for use in severe- } \\
\text { ly immunocompromised patient }\end{array}$ \\
\hline BAL GM & $\begin{array}{l}\text { Sensitivity: } 90 \%(77-96 \%) \\
\text { Specificity: } 94 \%(88-97 \%)\end{array}$ & $\begin{array}{l}\text { Positive LR: } 14.3(7.2-28.5) \\
\text { Negative LR: } 0.11(0.04-0.26)\end{array}$ & $\begin{array}{l}\text { Strong recommendation for use in } \\
\text { patients suspected of invasive fungal } \\
\text { diseases }\end{array}$ \\
\hline Serum BDG & $\begin{array}{l}\text { Sensitivity: } 73 \%(62-86 \%) \\
\text { Specificity: } 81 \% \text { (64-85\%) }\end{array}$ & $\begin{array}{l}\text { Positive LR: } 5.57 \text { (3.89-6.23) } \\
\text { Negative LR: } 0.34(0.12-0.48)\end{array}$ & \\
\hline $\begin{array}{l}\text { Single blood/serum } \\
\text { aspergillus PCR }\end{array}$ & $\begin{array}{l}\text { Sensitivity: 79.2\% (71.0-85.5\%) } \\
\text { Specificity: 79.6\% (69.9-86.6\%) }\end{array}$ & $\begin{array}{c}\text { Positive LR: } 3.8(2.6-5.7) \\
\text { Negative LR: } 0.26(0.18-0.36) \\
\text { PPV: } 42.8 \% \\
\text { NPV: } 95.1 \%\end{array}$ & $\begin{array}{l}\text { Strong recommendation for use in severe- } \\
\text { ly immunocompromised patients suspec- } \\
\text { ted of having IPA }\end{array}$ \\
\hline $\begin{array}{l}2 \text { blood/serum } \\
\text { aspergillus PCR }\end{array}$ & $\begin{array}{l}\text { Sensitivity: } 59.6 \%(40.7-76 \%) \\
\text { Specificity: } 95.1 \%(87-98.2 \%)\end{array}$ & $\begin{array}{c}\text { Positive LR: } 12.2(4.2-35.3) \\
\text { Negative LR: } 0.42(0.26-0.67) \\
\text { PPV: } 70.3 \% \\
\text { NPV }=92.4 \%\end{array}$ & \\
\hline BAL PCR & $\begin{array}{l}\text { Sensitivity: } 77 \%(62-87.6 \%) \\
\text { Specificity: } 93.5 \%(90.6-95.6 \%)\end{array}$ & $\begin{array}{l}\text { PPV: } 67.6 \% \\
\text { NPV: } 94.6 \%\end{array}$ & $\begin{array}{l}\text { Strong recommendation for use as a part } \\
\text { of evaluation in severely immunocompro- } \\
\text { mised patients suspected of having IPA }\end{array}$ \\
\hline
\end{tabular}

FN — false negative; FP — false positive; LR — likelihood ratio; NPV — negative predictive value; PPV — positive predictive value

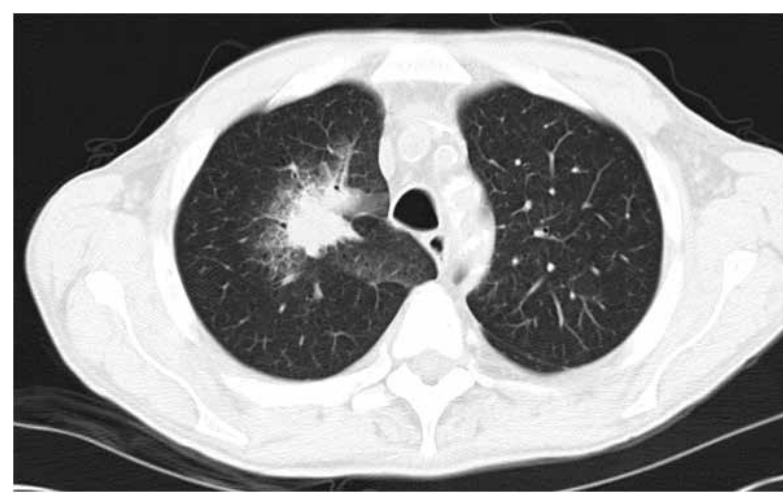

Figure 1. Chest CT image showing right upper lobe nodule with halo sign in a patient with IPA

\section{Treatment}

Early diagnosis and initiation of treatment for IPA greatly enhances chance of survival. However, as previously discussed, the clinical signs and symptoms can be non-specific and characteristic imaging findings are frequently absent and requires a high index of suspicion from physicians. Previously established definition for establishing diagnosis of proven or probably IPA by EORTC/MSG is time consuming, and clinicians should not delay therapy in at risk patients due to the rapid clinical progression and high mortality rate.

The Infectious Disease Society of America (IDSA) and the European Society for Clinical Microbiology and Infectious Diseases endorses prompt treatment of IPA with antifungal agents.
The drug of choice remains voriconazole, which has improved survival when compared with amphotericin B [38]. Typical treatment dose for IPA is $6 \mathrm{mg} / \mathrm{kg} \mathrm{IV}$ every 12 hours on day 1 , followed by $3 \mathrm{mg} / \mathrm{kg}$ every 12 hours. Transition to oral medication is appropriate once the patient is stable. Voriconazole is metabolized in the liver, and is both a substrate and an inhibitor of CYP2C19 and CYP3A4, and as a result can cause significant drug-drug interactions and thus requiring thorough medication review prior to initiation [15]. Major side effects include but are not limited to transient visual disturbances, dose-related hepatotoxicity, skin rash, reversible central and peripheral neurologic symptoms, and cardiovascular events. Therapeutic drug monitoring (TDM) has been utilized more commonly for monitoring efficacy and safety of treatment of IPA as patients often have multiple comorbidities or underlying illnesses that affect the drug absorption, metabolism, and clearance. Target serum trough level of $>1-1.5 \mu \mathrm{g} / \mathrm{mL}$ for most patients is considered adequate, and level should be checked 2-7 days after initiation of therapy, and further monitoring is based on patient's clinical status, disease severity, and concern for toxicity [15, 39].

A more recent development is the use of isavuconazole as the alternative primary treatment of choice in hematological patients, which showed to be non-inferior to voriconazole in the SECURE trial, with better tolerance $[38,40]$. Though less severe side effects, close attention should be 


\section{Table 2. Diagnostic criteria for invasive pulmonary aspergillosis}

\begin{tabular}{ll}
\hline \multicolumn{1}{c}{ Diagnosis } & \multicolumn{1}{c}{ Criteria } \\
\hline Proven & Sterile specimen obtained from a clinically or radiologically abnormal site with: \\
& - histo- or cytopathological evidence or direct microscopic identification of hyphae invading the lung tissue or \\
& - positive culture result for aspergillus \\
Probable & $\geq 1$ host factor and \\
& $\geq 1$ clinical criteria and \\
& $\geq 1$ mycological evidence (positive Aspergillus microscopy or culture from the sputum or BAL or positive antigen assay \\
& of GM or BDG \\
Possible & $\begin{array}{l}\text { Host factor and } \\
\text { clinical criteria consistent with the infection, no mycological evidence of aspergillus }\end{array}$ \\
\hline
\end{tabular}

BAL — Bronchoalveolar Lavage; BDG — b-D glucan; GM — galactomannan

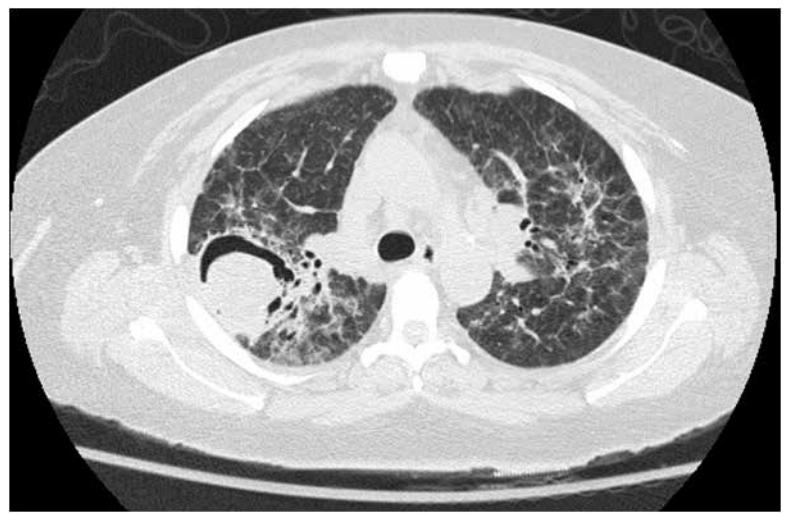

Figure 2. Chest CT image showing right upper lobe fungal ball in a patient with CPA

paid to co-administration of medications that are CYP3A4 substrates and/or inducers [15].

Amphotericin B and its lipid derivatives are appropriate agents for initial and salvage therapy when use of voriconazole is contraindicated or not tolerated. In the case of liver insufficiency, liposomal amphotericin B is the generally the therapy of choice.

Echinocandins such as caspofungin, and micafungin work by the noncompetitive inhibition of the synthesis of BDG [15]. Monotherapy with echinocandin is not generally recommended as initial treatment of IPA, however, it is an effective salvage therapy when used as monotherapy or in combination with other antifungal agents $[15,41,42]$. Specifically, synergistic effects have been described in multiple studies when used in combination with triazoles $[43,44]$. Their long half-life of $>10$ hours allows for daily dosing, and are relatively well tolerated, with few side effects, and few drug interactions. Echinocandins are available in parenteral form only.

There is no clear consensus regarding duration of therapy with antifungals, with the IDSA recommending minimum of 6-12 weeks depen- ding on clinical severity and disease course.

Surgical resection is reserved for patient with IPA and massive hemoptysis, or for localized IPA that's refractory to medical treatment [15].

Prophylaxis regimen for at risk populations during prolonged neutropenia is strongly recommended by IDSA. Posaconazole and voriconazole demonstrated superiority versus other triazoles [15]. Other agents used for prophylaxis include micafungin, amphotericin B and caspofungin.

Non-pharmacologic interventions (i.e. environmental strategies) such as the use of HEPA filters, with directed airflow in patient rooms, and positive pressure ventilation rooms for special at risk patients are utilized to prevent nosocomial pulmonary aspergillosis [45].

\section{Chronic pulmonary aspergillosis}

Chronic pulmonary aspergillosis (CPA) consists of a spectrum of disease patterns with varying degree of overlap in mostly immunocompetent individuals with underlying lung diseases, especially those with prior history of tuberculosis or non-tuberculous mycobacteria (NTM) [46]. It is estimated that approximately 3 million people suffer from CPA globally, with roughly 1.2 million develop CPA as a sequalae of pulmonary TB $[47,48]$. Other associated lung diseases include chronic obstructive pulmonary disease (COPD), emphysema, allergic bronchopulmonary aspergillosis (ABPA), asthma, cystic fibrosis, prior pneumothorax, prior treated lung cancer, sarcoidosis, ankylosing spondylitis, pneumoconiosis, and pneumonia [46, 49].

The most common form of CPA is chronic cavitary pulmonary aspergillosis (CCPA), which was previously named complex aspergilloma, in which one or more pulmonary cavities exist that can enlarge over time (Figure 2) [50]. When untreated, CCPA may progress to chro- 
nic fibrosing pulmonary aspergillosis (CFPA), with extensive pulmonary fibrosis in one or more lobes causing significant loss of lung function [50].

Aspergilloma and aspergillus nodule are less severe manifestations of CPA. Aspergilloma is a fungal ball in a pre-existing lung cavity and consists of aspergillus hyphae, fibrin, mucus, debris, inflammatory cells, and other degenerating blood and epithelial elements [13]. The term simple pulmonary aspergilloma is used when a single aspergillus fungal ball occupies a pulmonary cavity in a non-immunocompromised patient with no or minor symptoms, and have not progressed over at least 3 months [50]. The most common underlying cavitary pulmonary diseases involved is prior tuberculosis cavities. It is estimated that over $11-17 \%$ of reported aspergilloma are in patients with post-tuberculosis cavities. Other diseases include sarcoidosis, histoplasmosis, blastomycosis, AIDS, pneumonia, lung abscess, pulmonary fibrosis, asbestosis, rheumatoid nodules etc. [13].

An aspergillus nodule is defined as one or more nodules $(<3 \mathrm{~cm})$ that usually have no cavitation within. These are usually found incidentally on chest imaging.

Subacute invasive aspergillosis (SAIA), formally known as chronic necrotizing pulmonary aspergillosis, is comparably more rapidly progressive and locally destructive disease, and can be seen in mild to moderately immunosuppressed patients [50]. Diagnosis and treatment of SAIA is similar to that of IPA, but with a slower course of progression.

\section{Clinical presentations}

Most patients present with indolent, non-specific symptoms which cause delayed or missed diagnosis. Productive cough is the most common symptom. Other symptoms include shortness of breath, fatigue, weight loss, night sweats, fever and chest pain. Systemic symptoms are generally absent in patients with aspergilloma or aspergillus nodules. Hemoptysis is one of the typically presentations of CPA and occurs in $50-90 \%$ of patients with aspergilloma and can be life-threatening.

\section{Diagnosis}

The European Society for Clinical Microbiology and Infectious Diseases (ESCMID), the European Respiratory Society (ERS) and the European Confederation of Medical Mycology (ECMM) established diagnostic criteria for CPA in 2016 as the following: 1) one or more cavities with or without a fungal ball or nodules present on thoracic imaging for $\geq 3$ months; 2) direct evidence of Aspergillus infection or an immunological response to Aspergillus spp.; and 3) exclusion of alternative diagnoses (Table 3).

Similar criteria for CPA is published in the updated IDSA guideline in 2016 with the following criteria: patient with no or minimum immu-

Table 3. Diagnostic criteria for different manifestation of chronic pulmonary aspergillosis

\begin{tabular}{|c|c|c|c|}
\hline & Symptoms & Imaging finding & Confirmatory \\
\hline Aspergilloma & $\begin{array}{l}\text { Minimal or asympto- } \\
\text { matic }\end{array}$ & $\begin{array}{l}\text { Fungal ball with no radio- } \\
\text { graphic progression } \\
\text { over } \geq 3 \text { months }\end{array}$ & $\begin{array}{c}\text { Positive aspergillus IgG ( } \sim 90 \% \text { sensitive) or precipitins. } \\
\text { If negative, other evidence of aspergillus infection is } \\
\text { required }\end{array}$ \\
\hline CCPA & $\begin{array}{l}\text { Significant respiratory } \\
\text { and/or constitutional } \\
\text { symptoms }\end{array}$ & $\begin{array}{l}\text { One or more cavities } \pm \\
\text { aspergilloma with radio- } \\
\text { graphic progression } \\
\text { over } \geq 3 \text { months }\end{array}$ & $\begin{array}{l}\text { Any of the following once other diagnosis are excluded: } \\
\text { Positive aspergillus IgG or precipitins } \\
\text { Positive aspergillus antigen/DNA in respiratory fluids } \\
\text { Percutaneous or excision biopsy showing fungal hyphae/ } \\
\text { aspergillus spp. growing from a cavity }\end{array}$ \\
\hline CFPA & $\begin{array}{l}\text { Significant loss of lung } \\
\text { function }\end{array}$ & $\begin{array}{l}\text { Fibrosis with destruction } \\
\quad \text { of } \geq 2 \text { lung lobes }\end{array}$ & $\begin{array}{l}\text { Serological or microbiological evidence of aspergillus } \\
\qquad s p p .\end{array}$ \\
\hline Aspergillus nodule & Asymptomatic & $\begin{array}{l}\geq 1 \text { nodule } \\
\text { Usually }<3 \mathrm{~cm} \text {, may or } \\
\text { may not cavitate }\end{array}$ & Histological evidence of aspergillus \\
\hline SAIA & $\begin{array}{l}\text { Significant respiratory } \\
\text { and/or constitutional } \\
\text { symptoms }\end{array}$ & $\begin{array}{l}\text { Variable features including } \\
\text { cavitation, nodules, pro- } \\
\text { gressive consolidation with } \\
\text { radiographic progression } \\
\text { in 1-3 months }\end{array}$ & $\begin{array}{l}\text { Positive aspergillus IgG or precipitins or } \\
\text { Positive aspergillus antigen } \\
\text { Biopsy showing hyphae invasion of lung tissue }\end{array}$ \\
\hline
\end{tabular}

CCPA — chronic cavitary pulmonary aspergillosis; CFPA — chronic fibrosing pulmonary aspergillosis; SAIA — subacute invasive aspergillosis 
nocompromise; with varying degree of underlying pulmonary disease; who have at least 3 months of chronic pulmonary symptoms or chronic systemic illness or progressive radiographic abnormalities including cavitation, pleural thickening, pericavitary infiltrates, with or without fungal ball; and microbiologic evidence of aspergillosis infection [15]. The key difference is the ESCMID/ /ERS/ECMM guideline does not endorse detection of Aspergillus spp. in sputum nor serum galactomannan as diagnostic tests due to high rates of colonization and low sensitivity respectively. Though studies included patients with both CPA and IPA, the overall sensitive and specificity of utilizing BAL-GM aspergillus antigen assay (50 to $100 \%$ sensitivity, 77 to $100 \%$ specificity) is superior to that of serum-GM (9 to $67.5 \%$ sensitivity, 35 to $95 \%$ specificity) in diagnosing CPA [51-56]. Cut off level used also varied in these studies.

Based on clinical suspicion, plain chest radiograph is usually the initial imaging modality, and documented progression provides valuable information. CPA in general has a predilection for the upper lobes of the lungs [34]. CT scan provides more detailed evaluation of the lung parenchyma than plain films [57]. Many features of radiographic findings show significant overlap between the spectrum of diseases.

Simple aspergilloma appears as a solid oval opacity within a lung cavity, typically in the upper lobe, with a surrounding 'monod' or 'air crescent' as the mass is typically separated from the cavity wall forming an air space [58, 59]. The mass appears to move with changes in position. The radiographic appearance stays stable for months, in contrast to CCPA.

Hallmark of CCPA is consolidation, frequently in the upper lobes, that progress over time to cavitation and may lead to volume loss. Cavities vary in size, and cavity wall may be thin or irregularly thickened, with adjacent pleural thickening. CT of the lung allows for earlier diagnosis, showing thickening of the cavity wall and surrounding pleura.

CFPA result from untreated CCPA, with extensive pulmonary fibrosis involving one or more lobes, but commonly involving the whole hemithorax [50].

Aspergillus nodules can appear similar to malignancy, NTM, actinomycosis, or rheumatoid nodules, and can be difficult to differentiate based on radiologic imaging alone. Most are round, though spiculate edges are seen [50]. SAIA usually appear as a single area of consolidation in the upper lobes that progress to thin-walled cavity within days or weeks. There may be associated pleural thickening, fungus balls, pneumothorax, and pleural effusion seen [50].

\section{Treatment}

Treatment for CCPA is aimed at symptom improvement, to prevent and/or reduce hemoptysis and progression of lung fibrosis. Oral azole therapy with itraconazole $(200 \mathrm{mg}$ twice daily with therapeutic drug level monitoring) or voriconazole (150-200 mg twice daily with therapeutic drug level monitoring) is well-established first-line therapy [15, 60, 61]. Some may favor use of voriconazole in patients with heavy fungal load due to higher reported resistance rate to itraconazole, however, there has been little clinical evidence supporting this approach $[15,50]$. Few studies compare the efficacy of itraconazole verse voriconazole, and selection have largely been based on tolerability and cost. Tashiro et al. performed a retrospective analysis based on two randomized control trials comparing outcomes of using voriconazole or itraconazole as initial maintenance therapy. A total of 160 study patients were included, and they found no statistical difference in mortality between CPA patient treated with the two drugs, and no difference in rate of treatment discontinuation due to side effects [60].

As previously discussed, isavuconazole is a newer triazole approved for treatment of IPA. One study showed less adverse drug reaction in patient receiving isavuconazole. However, the study is limited by number, and the overall rate of discontinuation of therapy in both group were comparable [62]. Posaconazole (400 mg twice daily liquid form, or $300 \mathrm{mg}$ once daily tablet) is used as a third-line agent, with better tolerance in some cases, though efficacy studies are limited [63]. Failure on azole therapy and those with pan-azole resistance warrant intravenous therapy instead, and in acutely ill patients, initial treatment with intravenous antifungal therapy is appropriate. Corticosteroids should be avoided when possible unless otherwise indicated for underlying disease [15].

Hemoptysis frequently complicates CPA, and can be fatal. Oral tranexamic acid provides good control of hemoptysis in some cases. In cases of significant or life-threatening hemoptysis, bronchial artery embolization is indicated [15, 50]. Surgery can be considered in CCPA with severe hemoptysis or failed therapy, and studies have reported higher post-surgical complication rates in patient with CCPA [64-66]. 
Management options of Aspergilloma is dependent on clinical symptoms. For asymptomatic patients, close follow up with observation is indicated. Definitive treatment is surgical resection for patients with adequate pulmonary function [50]. However, surgery can have serious post-operative complications including hemorrhage, bronchopleural fistula and thus invasive methods are frequently contraindicated in patients due to underlying disease and poor pulmonary function [67]. Careful pre-operative assessment of respiratory status is necessary, and five-year survival after surgical treatment have been reported to be between $91-100 \%$ [64-66, 68] with low recurrence [69].

There is also no clear consensus regarding duration of treatment for CPA. For CCPA, a period of 4-6 months for initial treatment are generally recommended while monitoring for clinical response $[15,50]$. Relapse occur in over one third of treated cases [70], so more patients are left on long-term treatment, though the decision is individual based [50]. Common side effects were previously mentioned and often limit treatment, and drug resistance may develop.

Much like treatment duration, clinical tools to assess clinical response to treatment is also debated. Radiographic monitoring with low-dose CT without contrast or chest radiographs are often used. Decrease in cavity and pleural thickness and the loss of fungal ball on imaging paralleled clinical response the most [71].

The five year mortality rate for CCPA is between $40-60 \%$ - with variability in geographic location [60, 72]. Significant poor prognostic factors include previous infection with NTM, COPD, pleural involvement, bilateral cavitary disease, presence of aspergillomas, low BMI, hypoalbuminemia, old age, and breathlessness [72].

SAIA is considered as CPA, but treated similarly to acute invasive aspergillosis (IPA) due to its rapid progressive nature [50].

\section{Allergic bronchopulmonary aspergillosis}

Allergic bronchopulmonary aspergillosis (ABPA) is an inflammatory pulmonary disease characterized by hypersensitivity reaction to colonization of airway by aspergillus fumigatus in susceptible individuals, most of whom are patients with asthma and cystic fibrosis (CF) [73-75]. The term allergic bronchopulmonary mycosis is used to describe the immune sensitivity reactions to other fungal classes, though the predominant causative organism is Aspergillus fumigatus. ABPA was first described in literature in 1952 by Hinson et al., with most reported cases related to aspergillosis [76]. True prevalence is unclear due to variability in diagnosis, it is estimated that ABPA complicated up to $1-2 \%$ of asthma patients [77], and up to $15 \%$ of $\mathrm{CF}$ patients [78].

In susceptible individuals, Aspergillus fumigatus colonizes the airway, germinates, and develops mucus plugs that consist of hyphae and cause chronic allergic, inflammatory response that can ultimately lead to tissue and airway damage, bronchiectasis, and/or pulmonary fibrosis [79, 80].

The aspergillus spores release proteases and other antigens that trigger an immunologic response which involves an IgE-mediated type I hypersensitivity, an IgG-mediated type III hypersensitivity reaction, as well as that of Th2 CD4 T cell-mediated immune response by activation of cascade of inflammatory cytokines [81].

Multiple host genetic predisposition have been identified, including mutations in CF transmembrane conductance regulator (CFTR), HLA -DR 2 and 5 polymorphisms, IL-10 promoter polymorphisms and surfactant protein A polymorphism has been shown to be associated with increased susceptibility to ABPA, while HLA-DQ2 polymorphism have been shown to protect against ABPA [82-87].

\section{Clinical manifestations}

ABPA typically presents in patient with difficult to control asthma, however, the spectrum of disease presentation can be variable. Clinically, patients complain of symptoms including dyspnea, wheezing, thick, mucus-like productive cough, low-grade fever, malaise, and in some cases, hemoptysis [79, 88]. Clubbing can be seen in those with chronic bronchiectasis [89]. CF patient often present with worsening pulmonary function or new infiltrate on chest radiograph [90].

Plain chest radiographs may demonstrate either transient or permanent features consistent with ABPA including pulmonary opacities, "tramline shadows" indicating bronchial wall thickening, 'finger-in-glove' and 'toothpaste' shadows from mucoid impaction causing dilated bronchi [75]. HRCT allows better assessment of the structural pattern of ABPA and is often the imaging of choice. Central bronchiectasis with peripheral tapering has been referred to as a distinct feature of ABPA, though bronchiectasis is not present in all cases, and in some, peripheral bronchiectasis have been observed [75, 91]. Bronchiectasis on CT is characterized as 'signet ring' and 'string of pearls' [73]. Other findings on HRCT include mu- 
coid impaction, mosaic attenuation, centrilobular nodules and 'tree-in-bud' opacities. High-attenuation mucus (HAM) is when the mucus appears visually denser than the paraspinal skeletal muscle, which is a pathognomonic finding of ABPA [75, 89, 92].

\section{Diagnosis}

ABPA is likely underdiagnosed due to the variations in diagnostic criteria used globally [74]. Diagnostic criteria for ABPA was first proposed by Rosenberg et al. in 1977 [93-96]. Due to lack of consensus and lack of definitive diagnostic test, many other criteria have been proposed [94-96]. The ABPA working group of the International Society for Human and Animal Mycology (ISHAM) proposed an updated diagnostic and staging criteria in 2013 which included predisposing conditions of bronchial asthma or cystic fibrosis. Diagnostic features were divided into 'obligatory' and 'others'. Obligatory criteria included either positive type I Aspergillus skin test or elevated A. fumigatus specific IgE levels AND elevated total IgE levels of $>1000 \mathrm{IU} / \mathrm{mL}$. At least two of the following other criteria are required: 1) presence of precipitating or serum IgG antibodies against $A$. fumigatus; or 2) radiographic pulmonary opacities consistent with ABPA; or 3) total eosinophil count of $>500$ cells $/ \mu \mathrm{L}$ in steroid naïve patients [89]. A. fumigatus specific IgE levels is the best screening test for ABPA and is more sensitive than Aspergillus skin test. On the other hand, total serum IgE level is a sensitive indicator of response to therapy and relapse [89].

Those with uncontrolled asthma and positive aspergillus specific IgE levels but total IgE level of less that cut off of $1000 \mathrm{IU} / \mathrm{mL}$ and do not meet all criteria are classified as severe asthma associated with fungal sensitization (SAFS).

Bronchoscopy may reveal mucus plugs containing aggregates of eosinophils, fungal hyphae that is also pathognomonic for ABPA [88]. ABPA was previously categorized into five stages: 1) acute; 2) remission; 3) exacerbation; 4) corticosteroid dependent asthma; and 5) fibrotic lung disease [97]. The ISHAM working group added a stage zero for asymptomatic patients diagnosed with ABPA to allow for early recognition and potential treatment of such group [89].

Diagnosis of ABPA in CF patients poses additional challenges as many features of clinical presentation overlaps including radiographic lung infiltrates, increased cough and sputum productions, and mucus plugs, thus the diagnosis is often delayed which in turn has detrimental effects as early treatment can prevent and slow down irreversible lung damages. Due to these challenges, CF Foundation consensus conference in 2003 outlined guidelines specific for $\mathrm{CF}$ patients, with major difference being a lowered total serum IgE of $500 \mathrm{IU} / \mathrm{mL}$ used as part of the minimum criteria, and a high index of suspicion is needed in this patient population [78].

\section{Treatment}

Goal of ABPA treatment aims at control of symptoms, prevention of exacerbations, and reduction in progression of disease complications such as end-stage lung fibrosis or cavitary diseases. Treatment of asymptomatic ABPA patients is not clearly indicated, but close follow-up is recommended. Doubling of serum total IgE is an indicator for ABPA exacerbation. Oral glucocorticoids and antifungals have been used in the management of ABPAs, though decisions are based on patient response, disease severity, frequency of exacerbations, drug adverse effects, and any emerging antifungal resistance [15].

Systemic glucocorticoids are the main treatment in the management of acute ABPA with its anti-inflammatory and immunosuppressive effects [89]. The optimal dosing is not well-established. A common regimen starts with prednisone $0.5 \mathrm{mg} / \mathrm{kg}$ daily for 14 days followed by $0.5 \mathrm{mg} / \mathrm{kg}$ every other day with further tapering and discontinuation in 3 months [94]. There is a lack of clinical trials on the effectiveness of oral corticosteroids, but few case series have demonstrated responsiveness to prednisone with decline of total IgE, reduction in blood eosinophil counts, and clearance of radiographic lesions [91, 98]. One unblinded randomized trial showed that medium dose of oral glucocorticoids is just as effective as high-dose oral glucocorticoids in the treatment of acute ABPA with fewer side effects $[98,99]$. Clinical response to treatment is monitored every 1-2 months with marked decrease in total serum IgE levels of $>35 \%$, and resolution or improvement in radiographic infiltrates $[75,100]$. Despite their effectiveness, rates of relapse during tapering have been reported as high as 50\%, with some becoming steroid-dependent [91]. Chronic steroid use also puts patients at risk for adverse effects such as diabetes mellitus, hypertension, osteoporosis, and growth retardation.

Antifungal therapy including itraconazole and voriconazole have been used in the treatment of ABPA in select patients. They work by reducing the fungal burden thereby reducing the immune response. IDSA guideline recommend using both oral glucocorticoids and antifungals at the 
initiation of treatment, while the ABPA working group of ISHAM reserves use of antifungals for patients who is intolerant of corticosteroid taper, or those with recurrent exacerbations [15, 89]. As the initial treatment option for acute ABPA however, a randomized, unblinded trial found prednisolone to be more effective than itraconazole in achieving response $(100 \%$ vs $88 \%$; $\mathrm{P}=0.007$ ) , though itraconazole group had lower incidence of adverse reactions, and total decline in serum IgE, the time to first ABPA exacerbation and the number of ABPA exacerbations at follow -up were similar between the two groups [101]. When used in glucocorticoid-dependent ABPA, addition of itraconazole at $200 \mathrm{mg}$ twice daily showed positive responses including reduction in glucocorticoid dose, decrease in serum $\operatorname{IgE}$ levels, and improvement in either pulmonary function or exercise tolerance, or improvement in pulmonary infiltrates [102]. Duration of therapy of 16 weeks have been suggested based on past trials to avoid drug adverse effects [102, 103]. Cases of Cushing's syndrome have been reported in patient using both glucocorticoids and itraconazole, especially those treated with inhaled corticosteroids, likely owing to itraconazole's ability to impair glucocorticoid metabolism and thereby increasing plasma levels $[104,105]$. Newer antifungals include voriconazole, and posaconazole have shown promising clinical response, though more studies are needed to evaluate their use as currently only case series are available [106-108]. Lastly, nebulized amphotericin B was shown to reduce exacerbations in a case study involving 21 patients with ABPA complicating asthma [109].

Omalizumab, a human monoclonal antibody against IgE have been shown in case studies to be an effective adjunct therapy in asthmatic patients with refractory ABPA despite first-line treatments. A systemic review including 102 patients with ABPA most of whom had experienced treatment failure with systemic glucocorticoids or anti-fungal treatment showed addition of omalizumab decreased serum IgE level, decreased ABPA exacerbation $(2.74 \pm 2.31$ [1-12] to 0.38 $\pm 0.7[0-3], p<0.001$ ), and a steroid-sparing effect where $94 \%$ of patients either stopped or reduced prednisone dose [110]. No significant improvement in lung function was noted in this review [110]. Data supporting use of omalizumab use in ABPA patients with CF have been contradictory, and overall, more clinical trials are needed to provide definitive support for use of omalizumab in ABPA patients.

\section{Conclusion}

Pulmonary aspergillosis exists as a spectrum of pulmonary diseases from hypersensitivity reaction, to a slowly progressing destructive process, to acute invasive disease with high mortality rate. Early diagnosis and treatment are imperative to prognosis. High index of suspicion, knowledge of risk factors, and rapid diagnostic approach that includes the utilization of non-invasive tests are steps that help in early diagnosis. Recent advances in antifungal therapy has significantly improved outcome of these diseases. Further studies are still needed for more robust diagnostic tools and better tolerated therapies.

\section{Conflict of interest}

None declared.

\section{References:}

1. Kanj A, Abdallah N, Soubani AO. The spectrum of pulmonary aspergillosis. Respir Med. 2018; 141: 121-131, doi: 10.1016/j. rmed.2018.06.029, indexed in Pubmed: 30053957.

2. Dismukes WE. Clinical mycology. Oxford University Press, New York 2003.

3. Denning DW. Invasive aspergillosis. Clin Infect Dis. 1998; 26(4): 781-803; quiz 804, doi: 10.1086/513943, indexed in Pubmed: 9564455.

4. Taccone FS, Van den Abeele AM, Bulpa P, et al. Epidemiology of invasive aspergillosis in critically ill patients: clinical presentation, underlying conditions, and outcomes. Crit Care. 2015; 19: 7 , doi: 10.1186/s13054-014-0722-7, indexed in Pubmed: 25928694.

5. Khasawneh F, Mohamad T, Moughrabieh MK, et al. Isolation of Aspergillus in critically ill patients: a potential marker of poor outcome. J Crit Care. 2006; 21(4): 322-327, doi: 10.1016/j. jcrc.2006.03.006, indexed in Pubmed: 17175418.

6. Vandewoude KH, Blot SI, Benoit D, et al. Invasive aspergillosis in critically ill patients: attributable mortality and excesses in length of ICU stay and ventilator dependence. J Hosp Infect. 2004; 56(4): 269-276, doi: 10.1016/j.jhin.2004.01.006, indexed in Pubmed: 15066736.

7. Dragonetti G, Criscuolo M, Fianchi L, et al. Invasive aspergillosis in acute myeloid leukemia: Are we making progress in reducing mortality? Med Mycol. 2017; 55(1): 82-86, doi: 10.1093/mmy/myw114, indexed in Pubmed: 27915304.

8. Janssen JJ, Strack van Schijndel RJ, van der Poest Clement $\mathrm{EH}$, et al. Outcome of ICU treatment in invasive aspergillosis. Intensive Care Med. 1996; 22(12): 1315-1322, doi: 10.1007/ bf01709544, indexed in Pubmed: 8986479.

9. Kim A, Nicolau DP, Kuti JL. Hospital costs and outcomes among intravenous antifungal therapies for patients with invasive aspergillosis in the United States. Mycoses. 2011; 54(5): e301-e312, doi: 10.1111/j.1439-0507.2010.01903.x, indexed in Pubmed: 20557463.

10. Manuel RJ, Kibbler CC. The epidemiology and prevention of invasive aspergillosis. J Hosp Infect. 1998; 39(2): 95-109, doi: 10.1016/s0195-6701(98)90323-1, indexed in Pubmed: 9651854.

11. Groll A, Shah P, Mentzel C, et al. Trends in the postmortem epidemiology of invasive fungal infections at a University Hospital. Journal of Infection. 1996; 33(1): 23-32, doi: 10.1016/ s0163-4453(96)92700-0.

12. Lehrnbecher T, Frank C, Engels K, et al. Trends in the postmortem epidemiology of invasive fungal infections at a university hospital. J Infect. 2010; 61(3): 259-265, doi: 10.1016/j. jinf.2010.06.018, indexed in Pubmed: 20624423.

13. Chamilos G, Kontoyiannis DP. Aspergillus, candida, and other opportunistic mold infections of the lung, in fishman's pulmonary diseases and disorders. 5th edition. McGraw-Hill Education, New York 2015. 
14. Dagenais TRT, Keller NP. Pathogenesis of Aspergillus fumigatus in Invasive Aspergillosis. Clin Microbiol Rev. 2009; 22(3): 447-465, doi: 10.1128/CMR.00055-08, indexed in Pubmed: 19597008.

15. Patterson TF, Thompson GR, Denning DW, et al. Practice Guidelines for the Diagnosis and Management of Aspergillosis: 2016 Update by the Infectious Diseases Society of America. Clin Infect Dis. 2016; 63(4): e1-e60, doi: 10.1093/cid/ciw326, indexed in Pubmed: 27365388.

16. Segal BH. Aspergillosis. N Engl J Med. 2009; 360(18): 18701884, doi: 10.1056/NEJMra0808853, indexed in Pubmed: 19403905.

17. Gerson SL, Talbot GH, Hurwitz S, et al. Prolonged granulocytopenia: the major risk factor for invasive pulmonary aspergillosis in patients with acute leukemia. Ann Intern Med. 1984; 100(3): 345-351, doi: 10.7326/0003-4819-100-3-345, indexed in Pubmed: 6696356.

18. Alshabani K, Haq A, Miyakawa R, et al. Invasive pulmonary aspergillosis in patients with influenza infection: report of two cases and systematic review of the literature. Expert Rev Respir Med. 2015; 9(1): 89-96, doi: 10.1586/17476348.2015.996132, indexed in Pubmed: 25547335.

19. Shah MM, Hsiao EI, Kirsch CM, et al. Invasive pulmonary aspergillosis and influenza co-infection in immunocompetent hosts: case reports and review of the literature. Diagn Microbiol Infect Dis. 2018; 91(2): 147-152, doi: 10.1016/j.diagmicrobio.2018.01.014, indexed in Pubmed: 29454654.

20. Tejerina EE, Abril E, Padilla R, et al. Invasive aspergillosis in critically ill patients: An autopsy study. Mycoses. 2019; 62(8): 673-679, doi: 10.1111/myc.12927, indexed in Pubmed: 31177621.

21. Schauwvlieghe AF, Rijnders BJA, Philips N, et al. Invasive aspergillosis in patients admitted to the intensive care unit with severe influenza: a retrospective cohort study. Lancet Respir Med. 2018; 6(10): 782-792, doi: 10.1016/S22132600(18)30274-1, indexed in Pubmed: 30076119.

22. Gustot T, Maillart E, Bocci M, et al. Invasive aspergillosis in patients with severe alcoholic hepatitis. J Hepatol. 2014; 60(2): 267-274, doi: 10.1016/j.jhep.2013.09.011, indexed in Pubmed: 24055548.

23. Bennett JE, Dolin R, Blaser MJ, et al. Mandell, Douglas, and Bennett's Principles and Practice of Infectious Diseases. 4th edition. Elsevier/Saunders 2015: 2895-2908.

24. Meersseman W, Vandecasteele SJ, Wilmer A. Invasive aspergillosis in critically ill patients without malignancy. Am J Respir Crit Care Med. 2004; 170(6): 580-581, doi: 10.1164/rccm. 200401-093OC, indexed in Pubmed: 15229094.

25. Leeflang MM, Debets-Ossenkopp YJ, Visser CE, et al. Galactomannan detection for invasive aspergillosis in immunocompromized patients. Cochrane Database Syst Rev. 2008(4): CD007394, doi: 10.1002/14651858.CD007394, indexed in Pubmed: 18843747.

26. de Heer K, Gerritsen MG, Visser CE, et al. Galactomannan detection in broncho-alveolar lavage fluid for invasive aspergillosis in immunocompromised patients. Cochrane Database Syst Rev. 2019; 5: CD012399, doi: 10.1002/14651858.CD012399. pub2, indexed in Pubmed: 31107543.

27. Haydour Q, Hage CA, Carmona EM, et al. Diagnosis of fungal infections. A systematic review and meta-analysis supporting american thoracic society practice guideline. Ann Am Thorac Soc. 2019; 16(9): 1179-1188, doi: 10.1513/AnnalsATS. 201811-766OC, indexed in Pubmed: 31219341.

28. Lu Y, Chen YQ, Guo YL, et al. Lu, Y., , Diagnosis of Invasive Fungal Disease Using Serum (1 3)- -D-Glucan: A Bivariate Meta-Analysis. Internal Medicine, 2011. Intern Med. 2011; 50(22): 2783-2791, doi: 10.2169/internalmedicine.50.6175, indexed in Pubmed: 22082890.

29. Hou TY, Wang SH, Liang SX, et al. The screening performance of serum 1,3-beta-d-glucan in patients with invasive fungal diseases: a meta-analysis of prospective cohort studies. PLoS One. 2015; 10(7): e0131602, doi: 10.1371/journal. pone.0131602, indexed in Pubmed: 26146829.

30. Lamoth F, Cruciani M, Mengoli C, et al. -Glucan antigenemia assay for the diagnosis of invasive fungal infections in patients with hematological malignancies: a systematic review and meta-analysis of cohort studies from the Third European Conference on Infections in Leukemia (ECIL-3). Clin Infect Dis. 2012; 54(5): 633-643, doi: 10.1093/cid/cir897, indexed in Pubmed: 22198786.

31. Cruciani M, Mengoli C, Loeffler J, et al. Polymerase chain reaction blood tests for the diagnosis of invasive aspergillosis in immunocompromised people. Cochrane Database Syst Rev. 2015(9): CD009551, doi: 10.1002/14651858.CD009551.pub2, indexed in Pubmed: 26343815.

32. Avni T, Levy I, Sprecher H, et al. Diagnostic accuracy of PCR alone compared to galactomannan in bronchoalveolar lavage fluid for diagnosis of invasive pulmonary aspergillosis: a systematic review. J Clin Microbiol. 2012; 50(11): 3652-3658, doi: 10.1128/JCM.00942-12, indexed in Pubmed: 22952268.

33. Heng SC, Morrissey O, Chen SCA, et al. Utility of bronchoalveolar lavage fluid galactomannan alone or in combination with PCR for the diagnosis of invasive aspergillosis in adult hematology patients: a systematic review and meta-analysis. Crit Rev Microbiol. 2015; 41(1): 124-134, doi: 10.3109/1040841X.2013.804033, indexed in Pubmed: 23799871.

34. Prasad A, Agarwal K, Deepak D, et al. Pulmonary aspergillosis: what CT can offer before it is too late! J Clin Diagn Res. 2016 10(4): TE01-TE05, doi: 10.7860/JCDR/2016/17141.7684, indexed in Pubmed: 27190919.

35. Sanguinetti M. Diagnosis and treatment of invasive fungal infections: looking ahead. J Antimicrob Chemother, 2019. J Antimicrob Chemother. 2019; 74(Suppl. 2): ii27-ii37.

36. De Pauw B, Walsh TJ, Donnelly JP. Revised definitions of invasive fungal disease from the European Organization for Research and Treatment of Cancer/Invasive Fungal Infections Cooperative Group and the National Institute of Allergy and Infectious Diseases Mycoses Study Group (EORTC/MSG) Consensus Group. Clin Infect Dis. 2008; 46(12): 1813-1821, doi: 10.1086/588660, indexed in Pubmed: 18462102.

37. Hage CA, Carmona EM, Epelbaum O, et al. Microbiological laboratory testing in the diagnosis of fungal infections in pulmonary and critical care practice. An official american thoracic society clinical practice guideline. Am J Respir Crit Care Med. 2019; 200(5): 535-550, doi: 10.1164/rccm.201906-1185ST, indexed in Pubmed: 31469325.

38. Husain S, Camargo JF. Invasive Aspergillosis in solid-organ transplant recipients: Guidelines from the American Society of Transplantation Infectious Diseases Community of Practice. Clin Transplant. 2019; 33(9): e13544, doi: 10.1111/ctr.13544, indexed in Pubmed: 30900296.

39. Ullmann AJ, Aguado JM, Arikan-Akdagli S, et al. Diagnosis and management of Aspergillus diseases: executive summary of the 2017 ESCMID-ECMM-ERS guideline. Clinical Microbiology and Infection. 2018; 24: e1-e38, doi: 10.1016/j. cmi.2018.01.002.

40. Maertens J, Raad I, Marr K, et al. Isavuconazole versus voriconazole for primary treatment of invasive mould disease caused by Aspergillus and other filamentous fungi (SECU$\mathrm{RE})$ : a phase 3, randomised-controlled, non-inferiority trial. The Lancet. 2016; 387(10020): 760-769, doi: 10.1016/s01406736(15)01159-9.

41. Denning DW, Marr KA, Lau WM, et al. Micafungin (FK463), alone or in combination with other systemic antifungal agents, for the treatment of acute invasive aspergillosis. J Infect. 2006; 53(5): 337-349, doi: 10.1016/j.jinf.2006.03.003, indexed in Pubmed: 16678903.

42. Hiemenz JW, Raad II, Maertens JA, et al. Efficacy of caspofungin as salvage therapy for invasive aspergillosis compared to standard therapy in a historical cohort. Eur J Clin Microbiol Infect Dis. 2010; 29(11): 1387-1394, doi: 10.1007/s10096-0101013-0, indexed in Pubmed: 20703506.

43. Mukherjee PK, Sheehan DJ, Hitchcock CA, et al. Combination treatment of invasive fungal infections. Clin Microbiol Rev. 2005; 18(1): 163-194, doi: 10.1128/CMR.18.1.163-194.2005, indexed in Pubmed: 15653825.

44. Steinbach WJ. Newer combination antifungal therapies for invasive aspergillosis. Med Mycol. 2011; 49(Suppl. 1): S77-81.

45. Sehulster L, Chinn RYW. CDC, HICPAC. Guidelines for environmental infection control in health-care facilities. Re- 
commendations of CDC and the Healthcare Infection Control Practices Advisory Committee (HICPAC). MMWR Recomm Rep. 2003; 52(RR-10): 1-42, indexed in Pubmed: 12836624.

46. Smith NL, Denning DW. Underlying conditions in chronic pulmonary aspergillosis including simple aspergilloma. Eur Respir J. 2011; 37(4): 865-872, doi: 10.1183/09031936.00054810, indexed in Pubmed: 20595150.

47. Brown GD, Denning DW, Gow NAR, et al. Hidden killers: human fungal infections. Sci Transl Med. 2012; 4(165): 165rv13, doi: 10.1126/scitranslmed.3004404, indexed in Pubmed: 23253612.

48. Denning DW, Pleuvry A, Cole DC. Global burden of chronic pulmonary aspergillosis as a sequel to pulmonary tuberculosis. Bull World Health Organ. 2011; 89(12): 864-872, doi: 10.2471/ BLT.11.089441, indexed in Pubmed: 22271943.

49. Schweer KE, Bangard C, Hekmat K, et al. Chronic pulmonary aspergillosis. Mycoses. 2013; 57(5): 257-270, doi: 10.1111/ myc.12152.

50. Denning DW, Cadranel J, Beigelman-Aubry C, et al. Chronic pulmonary aspergillosis: rationale and clinical guidelines for diagnosis and management. Eur Respir J. 2016; 47(1): 4568, doi: 10.1183/13993003.00583-2015, indexed in Pubmed: 26699723.

51. Izumikawa K, Yamamoto Y, Mihara T, et al. Bronchoalveolar lavage galactomannan for the diagnosis of chronic pulmonary aspergillosis. Med Mycol. 2012; 50(8): 811-817, doi: 10.3109/13693786.2012.682228, indexed in Pubmed: 22568603.

52. Kitasato Y, Tao Y, Hoshino T, et al. Comparison of Aspergillus galactomannan antigen testing with a new cut-off index and Aspergillus precipitating antibody testing for the diagnosis of chronic pulmonary aspergillosis. Respirology. 2009; 14(5): 701-708, doi: 10.1111/j.1440-1843.2009.01548.x, indexed in Pubmed: 19659648.

53. Shin B, Koh WJ, Jeong BH, et al. Serum galactomannan antigen test for the diagnosis of chronic pulmonary aspergillosis. Journal of Infection. 2014; 68(5): 494-499, doi: 10.1016/j.jinf.2014.01.005.

54. Kono Y, Tsushima K, Yamaguchi K, et al. The utility of galactomannan antigen in the bronchial washing and serum for diagnosing pulmonary aspergillosis. Respir Med. 2013; 107(7): 1094-1100, doi: 10.1016/j.rmed.2013.04.007, indexed in Pubmed: 23632100 .

55. Sehgal IS, Dhooria S, Choudhary H, et al. Utility of serum and bronchoalveolar lavage fluid galactomannan in diagnosis of chronic pulmonary aspergillosis. J Clin Microbiol. 2019; 57(3), doi: 10.1128/JCM.01821-18, indexed in Pubmed: 30626661.

56. Nguyen MH, Jaber R, Leather HL, et al. Use of bronchoalveolar lavage to detect galactomannan for diagnosis of pulmonary aspergillosis among nonimmunocompromised hosts. J Clin Microbiol. 2007; 45(9): 2787-2792, doi: 10.1128/JCM.0071607, indexed in Pubmed: 17596367.

57. Desai SR, Hedayati V, Patel K, et al. Chronic aspergillosis of the lungs: unravelling the terminology and radiology. Eur Radiol. 2015; 25(10): 3100-3107, doi: 10.1007/s00330-015-3690-7, indexed in Pubmed: 25791639.

58. Franquet T, Müller N, Giménez A, et al. Spectrum of pulmonary aspergillosis: histologic, clinical, and radiologic findings. RadioGraphics. 2001; 21(4): 825-837, doi: 10.1148/radiographics.21.4.g01jl03825.

59. Davda S, Kowa XY, Aziz Z, et al. The development of pulmonary aspergillosis and its histologic, clinical, and radiologic manifestations. Clin Radiol. 2018; 73(11): 913-921, doi: 10.1016/j. crad.2018.06.017, indexed in Pubmed: 30075854.

60. Tashiro M, Takazono T, Saijo T, et al. Selection of oral antifungals for initial maintenance therapy in chronic pulmonary aspergillosis: A longitudinal analysis. Clin Infect Dis. 2019 [Epub ahead of print], doi: 10.1093/cid/ciz287, indexed in Pubmed: 30959519.

61. Barac A, Kosmidis C, Alastruey-Izquierdo A, et al. CPAnet. Chronic pulmonary aspergillosis update: A year in review. Med Mycol. 2019; 57(Suppl. 2): S104-S109, doi: 10.1093/ mmy/myy070, indexed in Pubmed: 30816975.

62. Bongomin F, Maguire N, Moore CB, et al. Isavuconazole and voriconazole for the treatment of chronic pulmonary aspergillosis: A retrospective comparison of rates of adverse events. Mycoses. 2019; 62(3): 217-222, doi: 10.1111/myc.12885, indexed in Pubmed: 30570179.
63. Felton TW, Baxter C, Moore CB, et al. Efficacy and safety of posaconazole for chronic pulmonary aspergillosis. Clin Infect Dis. 2010; 51(12): 1383-1391, doi: 10.1086/657306, indexed in Pubmed: 21054179.

64. Farid S, Mohamed S, Devbhandari M, et al. Results of surgery for chronic pulmonary Aspergillosis, optimal antifungal therapy and proposed high risk factors for recurrence--a National Centre's experience. J Cardiothorac Surg. 2013; 8: 180, doi: 10.1186/1749-8090-8-180, indexed in Pubmed: 23915502.

65. Muniappan A, Tapias LF, Butala P, et al. Surgical therapy of pulmonary aspergillomas: a 30-year North American experience. Ann Thorac Surg. 2014; 97(2): 432-438, doi: 10.1016/j. athoracsur.2013.10.050, indexed in Pubmed: 24365218.

66. Brik A, Salem AM, Kamal AlR, et al. Surgical outcome of pulmonary aspergilloma. Eur J Cardiothorac Surg. 2008; 34(4): 882-885, doi: 10.1016/j.ejcts.2008.06.049, indexed in Pubmed: 18701313.

67. He B, Wan C, Zhou W. Clinical profile and surgical outcome for different types of chronic pulmonary aspergillosis. Am J Trans Res. 2019; 11(6): 3671-3679, indexed in Pubmed: 31312378.

68. Akbari JG, Varma PK, Neema PK, et al. Clinical profile and surgical outcome for pulmonary aspergilloma: a single center experience. Ann Thorac Surg. 2005; 80(3): 23-27, doi: 10.1016/j.athoracsur.2005.03.078, indexed in Pubmed: 16122488.

69. Kasprzyk M, Pieczyński K, Mania K, et al. Surgical treatment for pulmonary aspergilloma - early and long-term results. Kardiochir Torakochirurgia Pol. 2017; 14(2): 99-103, doi 10.5114/kitp.2017.68738, indexed in Pubmed: 28747940.

70. Koyama K, Ohshima N, Suzuki J, et al. Recurrence of chronic pulmonary aspergillosis after discontinuation of maintenance treatment by antifungal triazoles. J Infect Chemother. 2014; 20(6): 375-379, doi: $10.1016 /$ j.jiac.2014.02.003, indexed in Pubmed: 24679654.

71. Godet C, Laurent F, Bergeron A, et al. CT imaging assessment of response to Treatment in chronic pulmonary aspergillosis. Chest. 2016; 150(1): 139-147, doi: 10.1016/j.chest.2016.02.640, indexed in Pubmed: 26905365.

72. Lowes D, Al-Shair K, Newton PJ, et al. Predictors of mortality in chronic pulmonary aspergillosis. Eur Respir J. 2017; 49(2), doi: 10.1183/13993003.01062-2016, indexed in Pubmed: 28179437.

73. Shah A, Panjabi C. Allergic bronchopulmonary aspergillosis: a perplexing clinical entity. Allergy Asthma Immunol Res. 2016; 8(4): 282-297, doi: 10.4168/aair.2016.8.4.282, indexed in Pubmed: 27126721.

74. Greenberger P, Patterson R. Allergic bronchopulmonary aspergillosis. J Allergy Clin Immunol Pract. 2014; 2(6): 703-708, doi: 10.1016/j.jaip.2014.08.007, indexed in Pubmed: 25439360

75. Agarwal R. Allergic bronchopulmonary aspergillosis. Chest. 2009; 135(3): 805-826, doi: 10.1378/chest.08-2586, indexed in Pubmed: 19265090

76. Hinson KFW, Moon AJ, Plummer NS. Broncho-pulmonary aspergillosis; a review and a report of eight new cases. Thorax. 1952; 7(4): 317-333, doi: 10.1136/thx.7.4.317, indexed in Pubmed: 13015523

77. Agarwal R, Aggarwal AN, Gupta D, et al. Aspergillus hypersensitivity and allergic bronchopulmonary aspergillosis in patients with bronchial asthma: systematic review and meta -analysis. Int J Tuberc Lung Dis. 2009; 13(8): 936-944, indexed in Pubmed: 19723372.

78. Stevens DA, Moss RB, Kurup VP, et al. Allergic bronchopulmonary aspergillosis in cystic fibrosis--state of the art: Cystic Fibrosis Foundation Consensus Conference. Clin Infect Dis. 2003; 37(Suppl. 3): S225-264, doi: 10.1086/376525, indexed in Pubmed: 12975753.

79. Chamilos G, Kontoyiannis DP. Aspergillus, candida, and other opportunistic mold infections of the lung. Fishman's Pulmonary Diseases and Disorders, e.a. Michael A. Grippi (ed.). McGraw-Hill, New York 2015.

80. Knutsen AP. Allergic bronchopulmonary aspergillosis. Clin Exp Allergy. 2015; 45(2): 298-299.

81. Moss RB. Pathophysiology and immunology of allergic bronchopulmonary aspergillosis. Med Mycol. 2005; 43(Suppl. 1): S203-S206, doi: 10.1080/13693780500052255, indexed in Pubmed: 16110813. 
82. Chauhan B, Santiago L, Kirschmann DA, et al. The association of HLA-DR alleles and T cell activation with allergic bronchopulmonary aspergillosis. J Immuno. 1997; 159(8): 40724076, indexed in Pubmed: 9378997.

83. Chauhan B, Knutsen Ap, Hutcheson PS, et al. T cell subsets, epitope mapping, and HLA-restriction in patients with allergic bronchopulmonary aspergillosis. J Clin Invest. 1996; 97(10): 2324-2331, doi: 10.1172/JCI118675, indexed in Pubmed: 8636413 .

84. Bains SN, Judson MA. Allergic bronchopulmonary aspergillosis. Clin Chest Med. 2012; 33(2): 265-281, doi: 10.1016/j. ccm.2012.02.003, indexed in Pubmed: 22640845.

85. Chauhan B, Santiago L, Hutcheson PS, et al. Evidence for the involvement of two different MHC class II regions in susceptibility or protection in allergic bronchopulmonary aspergillosis. J Allergy Clin Immunol. 2000; 106(4): 723-729, doi: 10.1067/ mai.2000.109913, indexed in Pubmed: 11031343.

86. Miller PW. Cystic fibrosis transmembrane conductance regulator (CFTR) gene mutations in allergic bronchopulmonary aspergillosis. Am J Hum Genet. 1996; 59(1): 45-51, indexed in Pubmed: 8659542.

87. Eaton TE, Weiner Miller P, Garrett JE, et al. Cystic fibrosis transmembrane conductance regulator gene mutations: do they play a role in the aetiology of allergic bronchopulmonary aspergillosis? Clin Exp Allergy. 2002; 32(5): 756-761, doi: 10.1046/j. 1365-2222.2002.01361.x, indexed in Pubmed: 11994102.

88. Chupp G. Allergic Bronchopulmonary Aspergillosis (Mycosis) and Severe Asthma with Fungal Sensitivity. Fishman's Pulmonary Diseases and Disorders. e.a. Michael A. Grippi (ed.). McGraw-Hill, New York 2015.

89. Agarwal R, Chakrabarti A, Shah A, et al. Allergic bronchopulmonary aspergillosis: review of literature and proposal of new diagnostic and classification criteria. Clin Exp Allergy. 2013; 43(8): 850-873, doi: 10.1111/cea.12141, indexed in Pubmed: 23889240.

90. Janahi IA, Rehman A, Al-Naimi AR. Allergic bronchopulmonary aspergillosis in patients with cystic fibrosis. Ann Thorac Med. 2017; 12(2): 74-82, doi: 10.4103/atm.ATM_231_16, indexed in Pubmed: 28469716.

91. Agarwal R, Gupta D, Aggarwal AN, et al. Allergic bronchopulmonary aspergillosis: lessons from 126 patients attending a chest clinic in north India. Chest. 2006; 130(2): 442-448, doi: 10.1378/chest.130.2.442, indexed in Pubmed: 16899843.

92. Agarwal R, Gupta D, Aggarwal AN. Clinical significance of hyperattenuating mucoid impaction in allergic bronchopulmonary aspergillosis: an analysis of 155 patients. Chest. 2007; 132(4): 1183-1190, doi: 10.1378/chest.07-0808, indexed in Pubmed: 17646221.

93. Rosenberg M, Patterson R, Mintzer R, et al. Clinical and immunologic criteria for the diagnosis of allergic bronchopulmonary aspergillosis. Ann Intern Med. 1977; 86(4): 405-414, doi: 10.7326/0003-4819-86-4-405, indexed in Pubmed: 848802.

94. Greenberger PA. Allergic bronchopulmonary aspergillosis. J Allergy Clin Immunol. 2002; 110(5): 685-692, doi: 10.1067/ mai.2002.130179, indexed in Pubmed: 12417875.

95. Greenberger PA. When to suspect and work up allergic bronchopulmonary aspergillosis. Ann Allergy Asthma Immunol. 2013; 111(1): 1-4, doi: 10.1016/j.anai.2013.04.014, indexed in Pubmed: 23806451.

96. Rodrigues J, Caruthers C, Azmeh R, et al. The spectrum of allergic fungal diseases of the upper and lower airways. Expert Rev Clin Immunol. 2016; 12(5): 531-550, doi: 10.1586/1744666X.2016.1142874, indexed in Pubmed: 26776889.

97. Patterson R, Greenberger PA, Radin RC, et al. Allergic bronchopulmonary aspergillosis: staging as an aid to management. Ann Intern Med. 1982; 96(3): 286-291, doi: 10.7326/00034819-96-3-286, indexed in Pubmed: 7059089.

98. Natarajan S, Subramanian P. Allergic bronchopulmonary aspergillosis: A clinical review of 24 patients: Are we right in frequent serologic monitoring? Ann Thorac Med. 2014; 9(4): 216-220, doi: 10.4103/1817-1737.140130, indexed in Pubmed: 25276240.
99. Agarwal R, Aggarwal AN, Dhooria S, et al. A randomised trial of glucocorticoids in acute-stage allergic bronchopulmonary aspergillosis complicating asthma. Eur Respir J. 2016; 47(2): 490-498, doi: 10.1183/13993003.01475-2015, indexed in Pubmed: 26585431 .

100.Ricketti A, Greenberger P, Patterson R. Serum IgE as an important aid in management of allergic bronchopulmonary aspergillosis. Journal of Allergy and Clinical Immunology. 1984; 74(1): 68-71, doi: 10.1016/0091-6749(84)90089-7.

101. Agarwal R, Dhooria S, Singh Sehgal I, et al. A randomized trial of itraconazole vs prednisolone in acute-stage allergic bronchopulmonary aspergillosis complicating asthma. Chest. 2018; 153(3): 656-664, doi: 10.1016/j.chest.2018.01.005, indexed in Pubmed: 29331473.

102.Stevens DA, Schwartz HJ, Lee JY, et al. A randomized trial of itraconazole in allergic bronchopulmonary aspergillosis. N Engl J Med. 2000; 342(11): 756-762, doi: 10.1056/ NEJM200003163421102, indexed in Pubmed: 10717010.

103. Wark PA, Hensley MJ, Saltos N, et al. Anti-inflammatory effect of itraconazole in stable allergic bronchopulmonary aspergillosis: a randomized controlled trial. J Allergy Clin Immunol. 2003; 111(5): 952-957, doi: 10.1067/mai.2003.1388, indexed in Pubmed: 12743557.

104.Lebrun-Vignes B, Archer VC, Diquet B, et al. Effect of itraconazole on the pharmacokinetics of prednisolone and methylprednisolone and cortisol secretion in healthy subjects. Br J Clin Pharmacol. 2001; 51(5): 443-450, doi: 10.1046/j.1365-2125.2001.01372.x, indexed in Pubmed: 11422002 .

105. Bolland MJ, Bagg W, Thomas MG, et al. Cushing's syndrome due to interaction between inhaled corticosteroids and itraconazole. Ann Pharmacother. 2004; 38(1): 46-49, doi: 10.1345/ aph.1D222, indexed in Pubmed: 14742792.

106. Hilliard T, Edwards S, Buchdahl R, et al. Voriconazole therapy in children with cystic fibrosis. J Cyst Fibros. 2005; 4(4): 215-220, doi: 10.1016/j.jcf.2005.05.019, indexed in Pubmed: 16243008 .

107. Glackin L, Leen G, Elnazir B, et al. Voriconazole in the treatment of allergic bronchopulmonary aspergillosis in cystic fibrosis. Ir Med J. 2009; 102(1): 29, indexed in Pubmed: 19284017.

108. Chishimba L, Niven RM, Cooley J, et al. Voriconazole and posaconazole improve asthma severity in allergic bronchopulmonary aspergillosis and severe asthma with fungal sensitization. J Asthma. 2012; 49(4): 423-433, doi: 10.3109/02770903.2012.662568, indexed in Pubmed: 22380765 .

109. Ram B, Aggarwal AN, Dhooria S, et al. A pilot randomized trial of nebulized amphotericin in patients with allergic bronchopulmonary aspergillosis. J Asthma. 2016; 53(5): 517-524, doi: 10.3109/02770903.2015.1127935, indexed in Pubmed: 26666774

110.Li JX, Fan LC, Li MH, et al. Beneficial effects of Omalizumab therapy in allergic bronchopulmonary aspergillosis: A synthesis review of published literature. Respir Med. 2017; 122: 33-42, doi: 10.1016/j.rmed.2016.11.019, indexed in Pubmed: 27993289 .

111.Denning DW, Park S, Lass-Florl C, et al. High-frequency triazole resistance found In nonculturable Aspergillus fumigatus from lungs of patients with chronic fungal disease. Clin Infect Dis. 2011; 52(9): 1123-1129, doi: 10.1093/cid/cir179, indexed in Pubmed: 21467016.

112.Page ID, Richardson MD, Denning DW. Comparison of six Aspergillus-specific IgG assays for the diagnosis of chronic pulmonary aspergillosis (CPA). J Infect. 2016; 72(2): 240249, doi: 10.1016/j.jinf.2015.11.003, indexed in Pubmed: 26680697.

113. Takazono t, Izumikawa K. Recent advances in chronic pulmonary aspergillosis. Frontiers in Microbiology. 2018; 9(1810), doi: 10.3389/fmicb.2018.01810. 